\title{
Surface film formation in vitro by infant and therapeutic surfactants: role of surfactant protein B
}

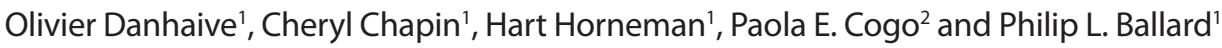

\begin{abstract}
BACKGROUND: Pulmonary surfactant provides an alveolar surface-active film that is critical for normal lung function. Our objective was to determine in vitro film formation properties of therapeutic and infant surfactants and the influence of surfactant protein (SP)-B content.
\end{abstract}

METHODS: We used a multiwell fluorescent assay measuring maximum phospholipid surface accumulation (Max), phospholipid concentration required for half-maximal film formation (1/2Max), and time for maximal accumulation (tMax).

RESULTS: Among five therapeutic surfactants, calfactant (highest SP-B content) had film formation values similar to natural surfactant, and addition of SP-B to beractant (lowest SP-B) normalized its Max value. Addition of budesonide to calfactant did not adversely affect film formation. In tracheal aspirates of preterm infants with evolving chronic lung disease, SP-B content correlated with $1 / 2$ Max and tMax values, and SP-B supplementation of SP-B-deficient infant surfactant restored normal film formation. Reconstitution of normal surfactant indicated a role for both SP-B and SP-C in film formation.

CONCLUSION: Film formation in vitro differs among therapeutic surfactants and is highly dependent on SP-B content in infant surfactant. The results support a critical role of SP-B for promoting surface film formation.

$\mathbf{P}$ ulmonary surfactant is a complex mixture of phospholipids (PLs) and proteins providing a surface-active film that allows expansion and prevents collapse of alveoli during respiratory cycles. Premature infants typically present with a transient deficiency of surfactant due to lung immaturity, leading to respiratory distress syndrome. A subset of these infants who evolve toward chronic lung disease develops surfactant dysfunction around the second week of life, associated with inadequate content of surfactant protein (SP)-B (1). SP-B is critical for intracellular packaging of surfactant in type 2 cell lamellar bodies, for its adsorption to the gas-liquid interface and the maintenance of an active surface film during respiration (2). Surfactant adsorption and surface activity can be inhibited in the presence of nonspecific proteins and other substances after lung injury (3).

Replacement surfactant therapy at birth decreases mortality and respiratory distress syndrome in preterm infants. Other indications include secondary surfactant deficiencies, such as worsening respiratory failure in the premature infant, meconium aspiration, pulmonary hemorrhage, or sepsis/pneumonia (4). Second-generation, synthetic surfactants containing various analogues of hydrophobic SPs are under development with the purpose of avoiding animal-based material, enhancing functional properties, and extending indications (5-7). Surfactants also have been tested as a drug delivery vehicle, providing efficient distribution throughout the lung and potentially reducing systemic exposure. One relevant clinical application is surfactant-mediated delivery of budesonide, a synthetic corticosteroid, which shows efficacy in preventing chronic lung disease in preterm infants with limited systemic effects and no observed adverse outcomes (8).

Surfactant function can be assessed in vitro using a variety of techniques including Langmuir-Whilhemy surface balance, captive bubble surfactometer, and pulsating bubble surfactometer (9). Recently, Ravasio et al. (10) described a technique for measuring PL surface film formation in multiwell plates containing fluorescence-labeled surfactant and a light quencher that allows high-throughput kinetic analyses at various concentrations of subphase surfactant; they established the principles of the assay, optimized the experimental conditions, and analyzed variables related to lipid composition in natural surfactant and synthetic lipid mixtures. In the current study, we tested the applicability of this new in vitro assay for both clinical samples and component-specific pharmacological testing, focusing on the role of the lipophilic surfactant proteins. We compared surface film formation properties of different therapeutic surfactants, assessed the role of endogenous SP-B content on film formation by surfactant samples from intubated premature infants, and tested the effects of supplemental SP-B and other additives. We found that therapeutic surfactants have different properties and that SP-B content critically affects PL surface film formation in this assay.

\section{RESULTS}

Parameters and Kinetics of Surface Film Formation In initial experiments (Supplementary Figure S1 and Supplementary Methods online), we further characterized technical aspects of the film formation assay developed by Ravasio 
et al. (10). We found that steady-state surface film PL content was equivalent with addition of labeled surfactant to the bottom of the well vs. application to the surface, that the presence of unlabeled surfactant at the surface markedly lowered signal from labeled, bottom-loaded surfactant, and that absence of rotational shaking did not alter results. These findings support a model of time-dependent adsorption, reorganization, and packing of PL at the surface with little recycling of lipid from the subphase.

In order to characterize dose-dependent film formation properties, we compared the time courses of serial dilutions ranging from 3.0 to $0.1 \mu \mathrm{g}$ PL over 30 or more shaking cycles (average duration: $33.5 \mathrm{~min}$ ). Surfactants typically show an initial unstable phase, where fluorescence peaks and/or decreases, then a plateau followed by a gradual decrease. With calfactant, fluorescence typically reached plateau between 5 and $15 \mathrm{~min}$ for 1.5 and $3.0 \mu \mathrm{g}$ PL (Figure 1a). The average fluorescence value during plateau, considered to represent maximal steadystate surface film formation, was designated maximal adsorption (Max). The time to reach Max was designated tMax (Figure 1a) and was typically assessed at $1.5 \mu \mathrm{g}$ PL. The PL concentration required for half-maximal interfacial accumulation $(1 / 2 \mathrm{Max})$ was considered to reflect concentration-dependent efficiency of film formation (Figure 1b). For each experiment,

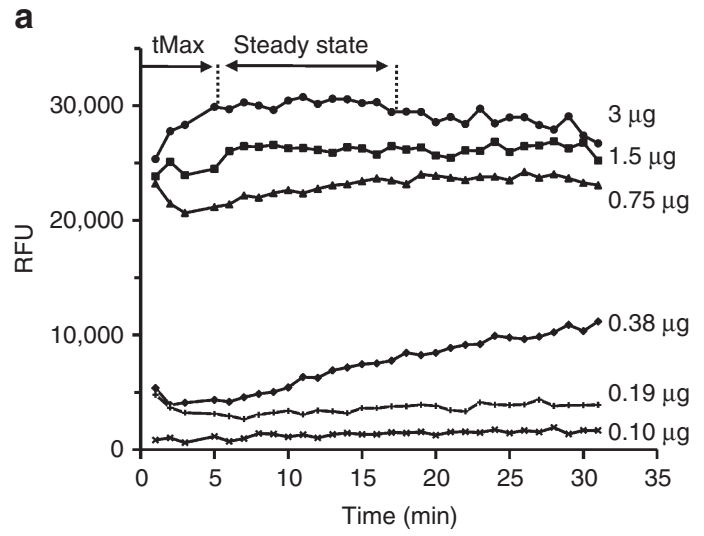

calfactant was chosen as internal control because its PL and SP-B contents are similar to natural surfactants $(11,12)$ and are constant between lots. Calfactant was very similar to freshly lavaged rat surfactant (Figure 1c) in terms of Max, $1 / 2 \mathrm{Max}$, and tMax $(5.3 \pm 1.9$ and $4.3 \pm 1.8 \mathrm{~min})$.

\section{Properties of Therapeutic Surfactants}

We compared surface film formation properties of calfactant (Infasurf), beractant (Survanta), poractant alfa (Curosurf), colfosceryl palmitate (Exosurf), and lucinactant (Surfaxin). There was an approximately twofold range in values for Max (Figure 2a), 1/2Max (Figure 2b), and tMax (Figure 2c) for the five surfactants, with calfactant demonstrating the best overall film formation properties. Next, we tested the effect of exogenous SP-B supplementation of beractant, which has a much lower SP-B content than calfactant ( 0.04 vs. $0.7-0.9 \%$ PL) $(11,13)$. Beractant Max increased after $1.25 \%$ SP-B supplementation; tMax also improved approximately twofold, but there was no change in $1 / 2$ Max (Figure $2 d$ ).

\section{Properties of Infant Endogenous Surfactant}

Surfactant of premature infants with respiratory failure is often deficient in SP-B and SP-C (13). We examined the

\section{b}

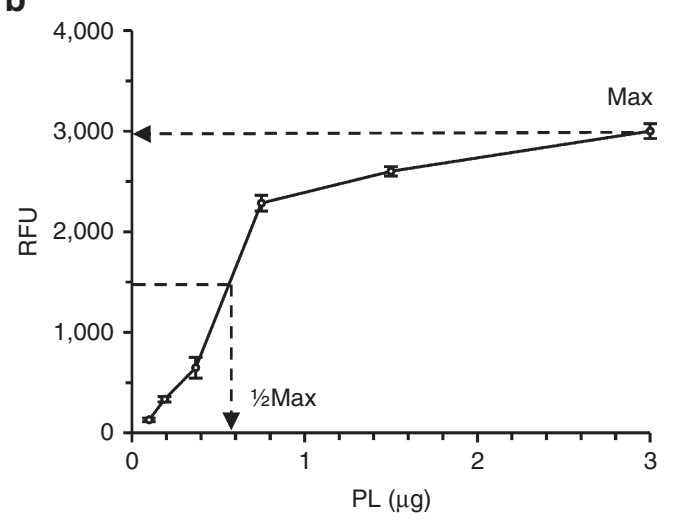

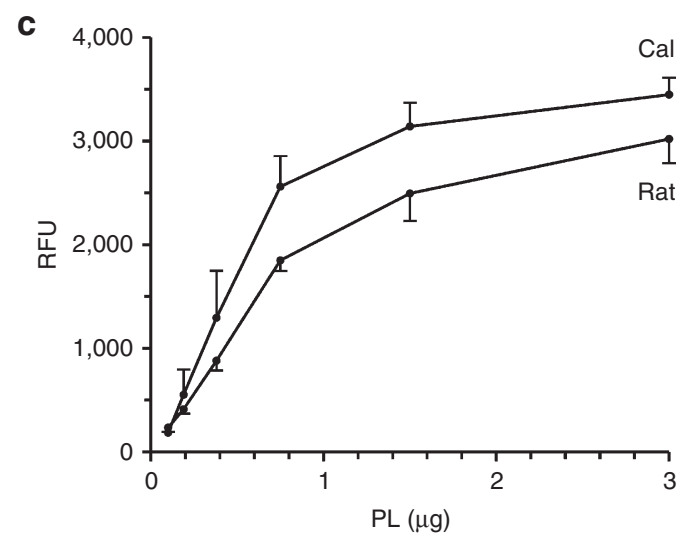

Figure 1. Properties of film formation assay. (a) Representative time course of surface fluorescence for serial dilutions of calfactant (3.0-0.1 $\mu \mathrm{g}$ PL). The average fluorescence at steady state (Max) is $30,160 \pm 1,270 \mathrm{RFU}$, and the time needed to reach Max (tMax) is $5.3 \pm 1.5 \mathrm{~min}$ for a $3 \mu \mathrm{g}$ calfactant load. (b) Fluorescence as a function of PL concentration: Max values are obtained at the highest PL concentrations ( $3.0 \mu \mathrm{g})$. The PL concentration required to achieve half-maximal $(1 / 2 \mathrm{Max}, 0.52 \pm 0.04)$ is shown by dashed lines. (c) Comparison of calfactant and rat bronchoalveolar lavage surfactant in a representative experiment in triplicate shows similar Max (34,480 $\pm 1,640$ and 30,200 $\pm 2,330 \mathrm{RFU}$, respectively) and 1/2Max (0.54 \pm 0.12 and $0.58 \pm 0.08)$. Data are mean \pm SEM. PL, phospholipid; RFU, relative fluorescence units. 
a

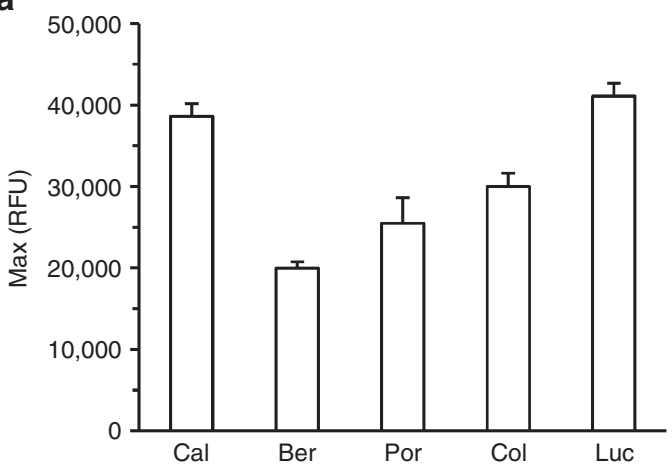

C

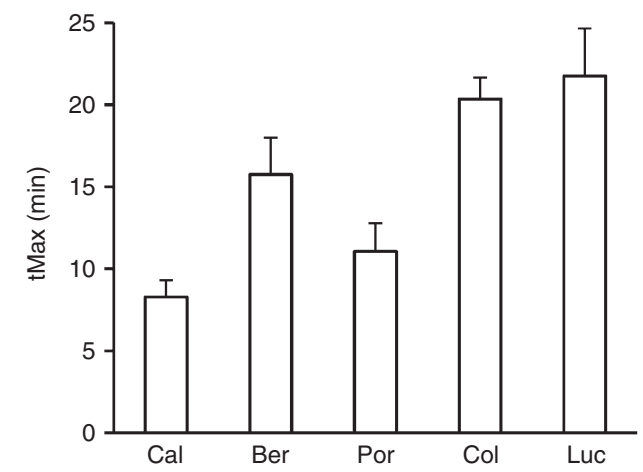

b

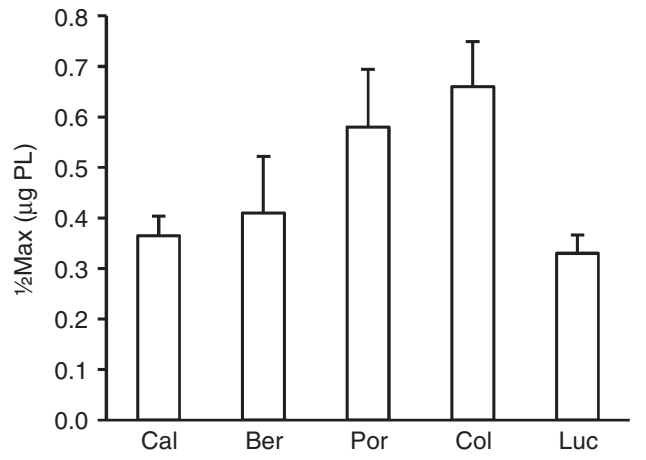

d

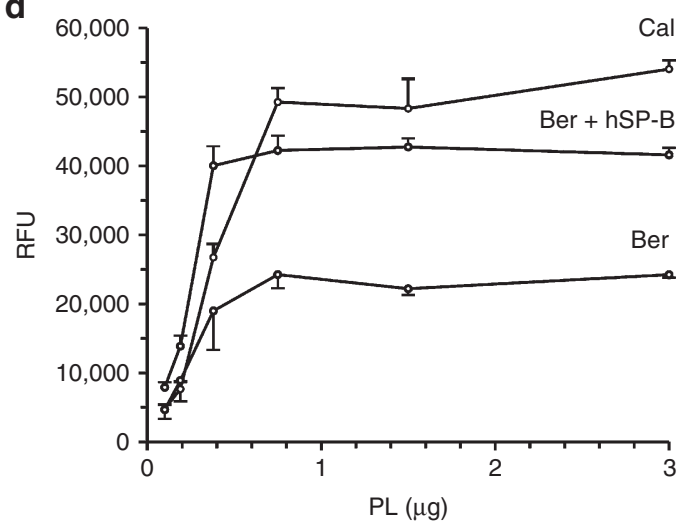

Figure 2. Film formation properties of therapeutic surfactants. (a) Max at $1.5 \mu \mathrm{g}$ PL for calfactant (Cal, 38,620 $\pm 1,550$ RFU) is higher than that for beractant (Ber, 19,990 \pm 770 ), poractant (Por, 25,480 $\pm 3,140$ ), and colfosceryl palmitate (Col, 29,990 $\pm 1,630)$, and similar to lucinactant (Luc, 41,080 $\pm 1,580)$; Max for Ber is lower than that for Col and Luc; Max for Col is lower than that for Luc $(P<0.0001)$. (b) $1 / 2$ Max values are lower for Cal $(0.37 \pm 0.04)$ than that for Por $(0.68 \pm 0.09)$ and $\mathrm{Col}(0.74 \pm 0.06) ; 1 / 2 \mathrm{Max}$ for Luc $(0.33 \pm 0.04)$ is lower than that for Por and Col; $1 / 2 \mathrm{Max}$ for Ber is lower than that for Por and Col $(P=0.03)$. (c) tMax for Cal (8.3 $\pm 1.0 \mathrm{~min})$ is lower than that for Ber (15.8 \pm 2.2$)$, Col (20.4 \pm 1.3$)$, and Luc (21.6 \pm 2.9$)$; tMax for Luc is higher than that for Por $(P<0.0001)$. (d) Addition of SP-B 1.2\% PL to beractant, increasing SP-B content from 0.05 to $1.25 \%$ PL, increases Max $(41,610 \pm 450$ vs. $24,250 \pm 1,030 \mathrm{RFU} ; P<0.001)$ to a level similar to that for calfactant. $1 / 2$ Max values were not affected by SP-B $(0.24 \pm 0.04$ and $0.22 \pm 0.01 \mu \mathrm{g}$ PL) but are lower than that for calfactant $(0.44 \pm 0.02 ; P<0.001)$. For $\mathbf{a}-\mathbf{c}$, data are mean \pm SEM for four experiments with triplicates ( $P$ by ANOVA with Bonferroni-Dunn post hoc test). For $\mathbf{d}$, data are mean \pm SEM for triplicates in a representative experiment; $P$ by paired Student's $t$-test. PL, phospholipid; RFU, relative fluorescence units.

association between Max, tMax, and 1/2Max and SP-B content (range: $0.02-1.02 \% \mathrm{PL}$ ) in surfactant samples of premature infants $(1,14)$. There were nonsignificant negative correlations of SP-B/PL content with Max (Figure 3a) and tMax (Figure 3c), and a highly significant $(P<0.0001)$ negative correlation with $1 / 2 \mathrm{Max}$ (Figure $3 \mathrm{~b}$ ). Total protein content of the surfactant pellet (range: $9-57 \%$ PL) showed an opposite, significant correlation with $1 / 2 \mathrm{Max}$ (Figure 3d), suggesting that serum and cellular proteins present in the alveolus inhibit surface film formation. The significant correlation of $1 / 2 \mathrm{Max}$ with the SP-B/protein ratio (Figure 3e) and a similar trend for tMax (Figure 3f)-indicates that the degree of inhibition by extraneous proteins depends on the relative amount of SP-B.

To further analyze the influence of SP-B, we added SP-B to a pooled tracheal aspirate (TA) sample with low endogenous SP-B content $(0.05 \% \mathrm{PL})$ from the same source $(1,14)$. Max values were not altered by SP-B up to 5\% PL (Figure 4a), while $1 / 2 \operatorname{Max}$ (Figure $4 \mathrm{~b}$ ) and tMax (Figure 4c) were strikingly reduced in a dose-dependent manner with a maximal effect reached around physiological concentrations $(0.8 \%$ PL for $1 / 2$ Max, $>1.3 \%$ for tMax).
Using TA from a term infant without lung disease, we isolated surfactant lipophilic components and tested film formation with PL alone and after partial reconstitution with either SP-B and/or SP-C fractions (Figure 5). Whereas PL alone showed poor film formation capacity regardless of the concentration, addition of either SP-B or SP-C to PL led to a greater than twofold increase in Max and a 40\% reduction in $1 / 2$ Max. Combining SP-B and SP-C resulted in an additive, sixfold increase in Max, whereas $1 / 2$ Max values did not further improve.

\section{Effect of Budesonide}

Supplementation of calfactant with the corticosteroid budesonide resulted in a dose-dependent increase of Max values for concentrations $\geq 1 \%$ (Figure $6 \mathrm{~b}$ ) with no effect on $1 / 2$ Max (Figure 6a) or tMax (data not shown). In order to rule out budesonide autofluorescence or direct budesonide binding of BODIPY, the fluoresecent dye used in this assay (see Methods), we repeated dose-response experiments using budesonide-BODIPY in absence of calfactant or budesonidecalfactant in absence of BODIPY and obtained no surface fluorescence (data not shown). These results indicate that 
a
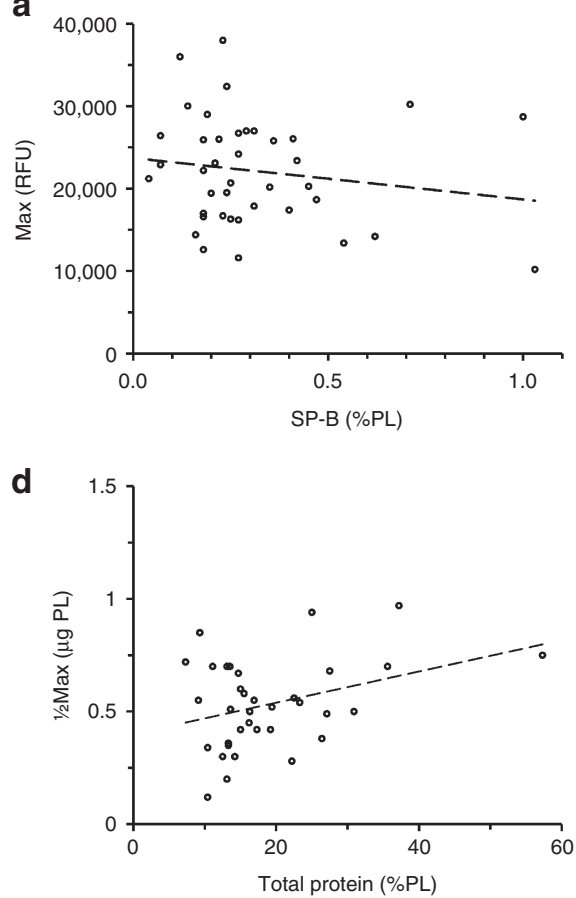

b

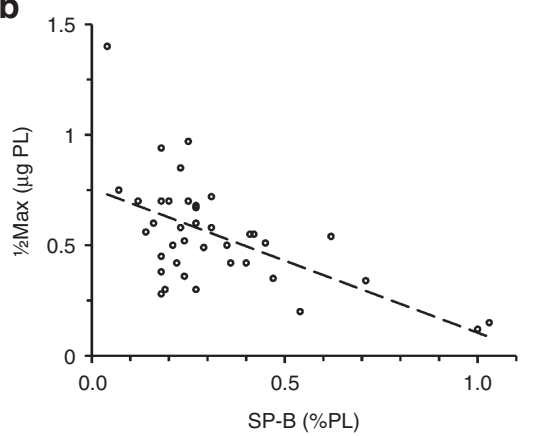

e

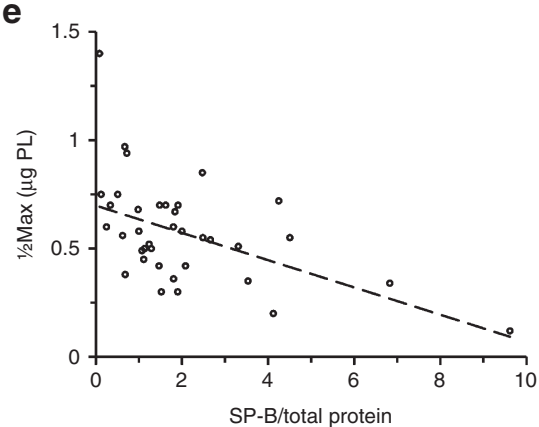

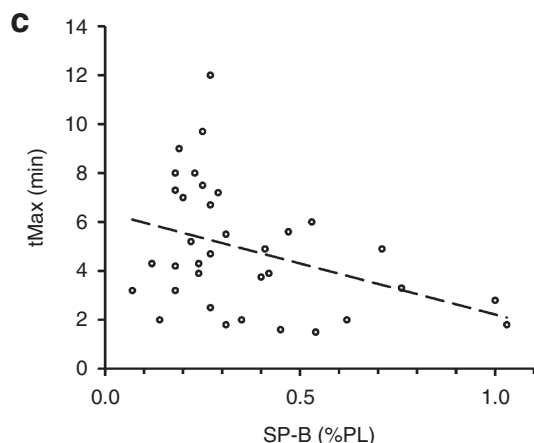

f

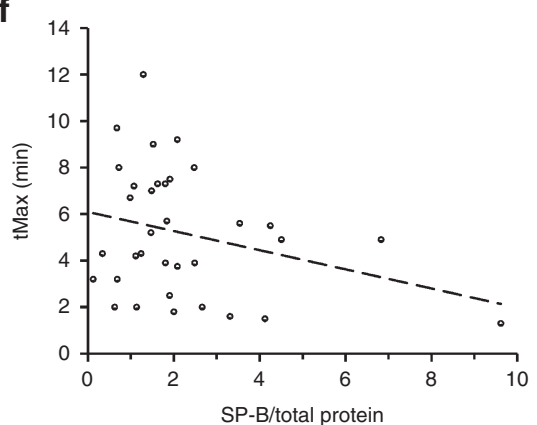

Figure 3. Film formation properties of surfactant isolated from premature infants. (a) Max values as a function of SP-B/PL concentration ( $r=-0.18$; $P=0.27$ ). (b) $1 / 2$ Max values as a function of SP-B concentration for the same samples $(r=-0.62 ; P=0.0001)$. (c) tMax values as a function of SP-B concentration $(r=-0.18 ; P=0.28)$. (d) $1 / 2$ Max values as a function of total protein content of the same surfactant samples $(r=0.42 ; P=0.008)$. (e) $1 / 2$ Max values as a function of the SP-B/total protein ratio $(r=-0.51 ; P=0.001)$. (f) tMax values as a function of the SP-B/total protein ratio $(r=-0.28$; $P=0.10$ ). SP-B specifically improves film formation efficiency, while nonspecific alveolar proteins isolated with surfactant are inhibitory. Data are individual values for 40 samples assayed in triplicate. PL, phospholipid; RFU, relative fluorescence units; SP, surfactant protein.

budesonide supplementation does not interfere with film formation in vitro. By contrast, addition of albumin, a known inhibitor of surfactant function, to calfactant at $33 \%$ PL significantly increased the $1 / 2$ Max value (data not shown), confirming published results (10).

\section{DISCUSSION}

Surfactant contains over 50 different lipids and a variety of proteins. Several studies have shown the critical role of the dominant PL, dipalmitoylphosphatidylcholine, and of the two hydrophobic proteins SP-B and SP-C for surface adsorption and activity $(15,16)$. In this article, using a high-throughput multiwell fluorescence assay, we demonstrated the dose-dependent effect of endogenous and supplemental SP-B in infant surfactant surface film formation and efficiency, and we confirmed that budesonide, another additive with no known surface activity, does not impair film formation in vitro.

PLs alone do not support fast adsorption and low surface tension properties under compression/expansion cycles (17). Addition of SP-B and/or SP-C significantly improves surface tension properties for a wide range of compressions and over repeated respiratory cycles by allowing tri-dimensional transformation of the surface film into a complex subphase reservoir (16). SP-B is thought to play an essential role in this process by promoting insertion of subphase PL vesicles into a lipid monolayer during the alveolar surface expansion associated with inspiration, whereas SP-C may be essential in maintaining the interface monolayer integrity at the highest compression states during the expiratory phase (17). Our findings of an inverse relationship between SP-B content and $1 / 2 \mathrm{Max}$ in the human TA samples are consistent with published minimum surface tension data (1) and support a dose-dependent role for SP-B in low PL conditions.

The microplate fluorescent assay provides a direct, dynamic readout of surface film formation and permits the simultaneous analysis of multiple samples at physiological PL concentrations and temperature. This technique was developed using animal surfactant and PL mixtures and was subsequently used to test in vitro inhibition of animal surfactant by meconium (18). We adapted the assay to measure film formation properties of surfactant from preterm infants with respiratory failure. The three parameters Max, $1 / 2 \operatorname{Max}$, and tMax reflect distinct functional features of surfactants in surface film formation and homeostasis. Max, by measuring the total PL amount contributing to the surface film and immediate subphase at equilibrium, likely reflects film formation efficacy and organization (10). $1 / 2 \mathrm{Max}$ measures film formation with lower PL concentrations, a proxy for surfactant efficiency. tMax is a more qualitative parameter that likely reflects both adsorption and film organization.

Using neonatal samples with low SP-B, we showed that supplementation with exogenous SP-B did not change Max but decreased $1 / 2$ Max values in a dose-dependent manner up to $1 \%$, which is within the physiologic concentration range in native surfactants (13); these data support the hypothesis that SP-B at physiological concentration optimizes film formation 


\section{Articles | Danhaive et al.}
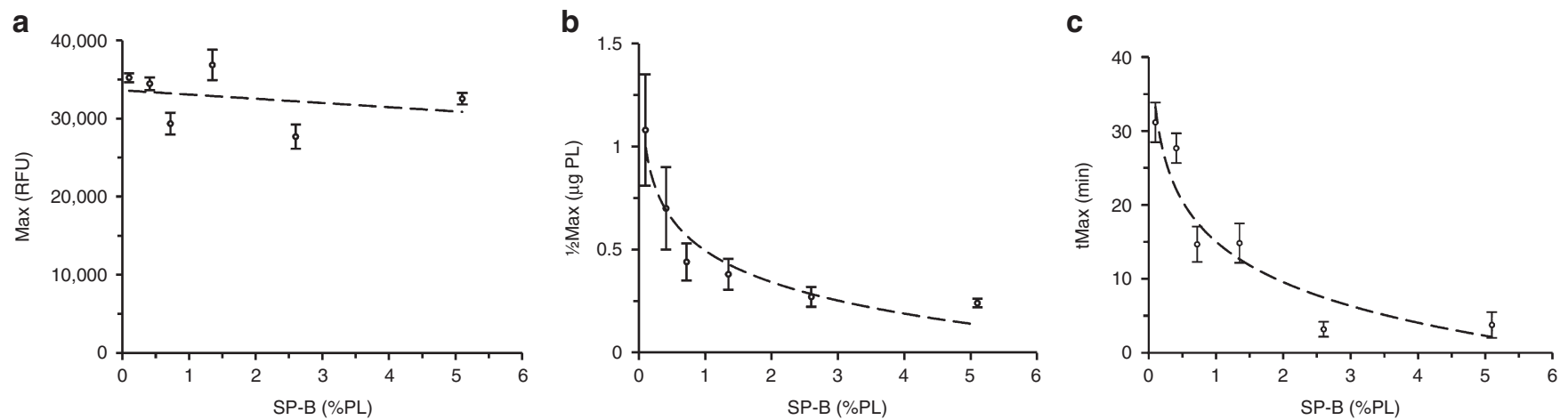

Figure 4. Supplemental SP-B effect on infant surfactant. Increasing amounts of purified human SP-B were added to pooled surfactant from 10 tracheal aspirate samples with low endogenous SP-B concentration $(0.05 \% \mathrm{PL})$. (a) Max values were not significantly affected by SP-B (mean \pm SEM for triplicates, $r=-0.29 ; P=0.59$ ). (b) $1 / 2$ Max values decrease in a curvilinear manner with increasing SP-B content. (c) tMax values similarly decrease in a curvilinear manner. PL, phospholipid; RFU, relative fluorescence units; SP, surfactant protein.

for lower PL concentrations in the alveolar fluid lining, as suggested by others using epifluorescence microscopy (19). Our experiment with reconstitution of PL, SP-B, and SP-C components showed a complementary role for SP-B and SP-C that will be investigated in future experiments.

Therapeutic surfactants differed in terms of Max and $1 / 2$ Max. Although species-related differences in PL and protein composition may exist, the observed differences likely depend on extraction procedures and formulation (see Supplementary Methods online). Calfactant is prepared by lipid extraction from calf lung lavage, whereas organic solvent extraction of lung tissue homogenate is used for poractant alfa (swine) and beractant (bovine), a process more likely to alter the PL/SP composition and to include nonspecific contaminants (11). The Max and $1 / 2$ Max adsorption values for calfactant were similar to those for natural surfactant, which presumably reflects the similarity in PL composition and SP-B and SP-C contents. By comparison, both beractant and poractant have lower SP-B contents, 0.04 and $0.26 \%$ PL, respectively (11). In contrast to infant samples, beractant's lower SP-B content did not translate into higher $1 / 2$ Max values but rather a low Max that increased with SP-B supplementation, confirming published observations (20); this response to SP-B may relate to the PL composition. Colfosceryl palmitate, a first-generation protein-free synthetic surfactant, had relatively high Max values but a longer tMax in this assay, in contrast to previous results indicating rapid adsorption with the pulsating bubble surfactometer (21). Lucinactant, a second-generation synthetic surfactant shown to be efficacious and cost effective (22), had Max and $1 / 2 \mathrm{Max}$ values comparable to calfactant but a significantly longer tMax. It is important to note that all of these surfactants demonstrate clinical benefit for infants, and thus, our in vitro results should be interpreted with caution regarding relative clinical efficacy.

We showed that budesonide addition to calfactant has no effect on film formation properties up to a threshold of $1 \%$, confirming results for surface properties by others with the pulsating bubble surfactometer method (8). At 5\% concentration, budesonide actually increased film formation. Budesonide has a sterol ring chemical structure that may act similarly to cholesterol, as a condenser of phosphatidylcholine

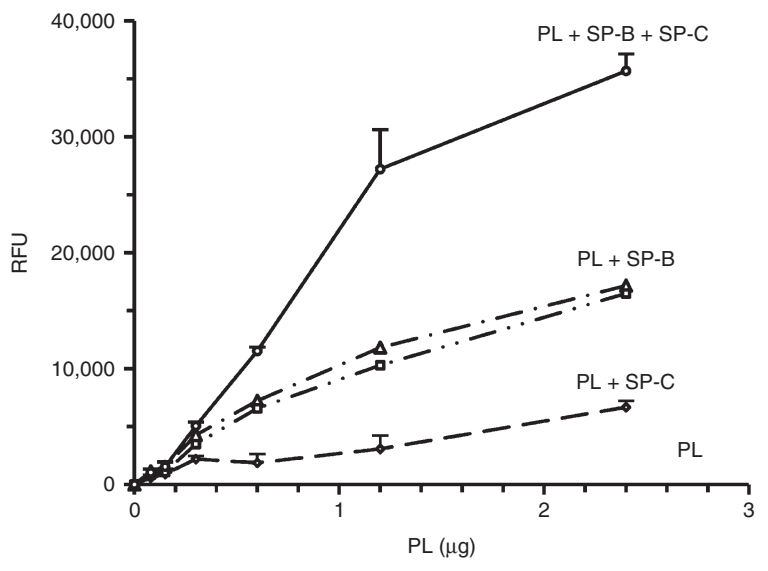

Figure 5. Film formation properties for PL without and with hydrophobic SPs. Surfactant from a term neonate without lung disease was separated into PL, SP-B, and SP-C fractions. PL alone had a low Max (6670 70 RFU); addition of either SP-B or SP-C fractions (to their original concentrations) increased Max fluorescence by approximately twofold and reduced the $1 / 2$ Max value by $40 \%$. Addition of both SP-B and SP-C fractions further increased the Max. Mean \pm SEM for triplicates. PL, phospholipid; RFU, relative fluorescence units; SP, surfactant protein.

monolayers, enhancing transfer and spreading at the surface (23). Other investigators using the pulsating bubble surfactometer observed significantly higher surface tension values with $20 \%$ budesonide (24), an adverse effect possibly related to budesonide interference with phosphatidylcholine compressibility and subphase formation. Our observations support the safety, with regard to surfactant function, of low-concentration budesonide-calfactant mixtures for intratracheal delivery.

While this assay is well suited for cohort-based research with high-throughput film formation measurements of multiple samples, an important limitation is recognized. Obvious differences exist between film formation in this in vitro assay and in vivo. In the alveolus, the fluid lining is very thin $(\sim 0.1 \mathrm{~mm})$, and surfactant concentration is very high $(\sim 150 \mathrm{mg} / \mathrm{ml})(25)$, allowing film formation to take place in a fraction of second. This assay, which has a much larger scale and lacks compression/expansion cycles, does not provide information on lowering of surface tension. Nevertheless, potential applications for 


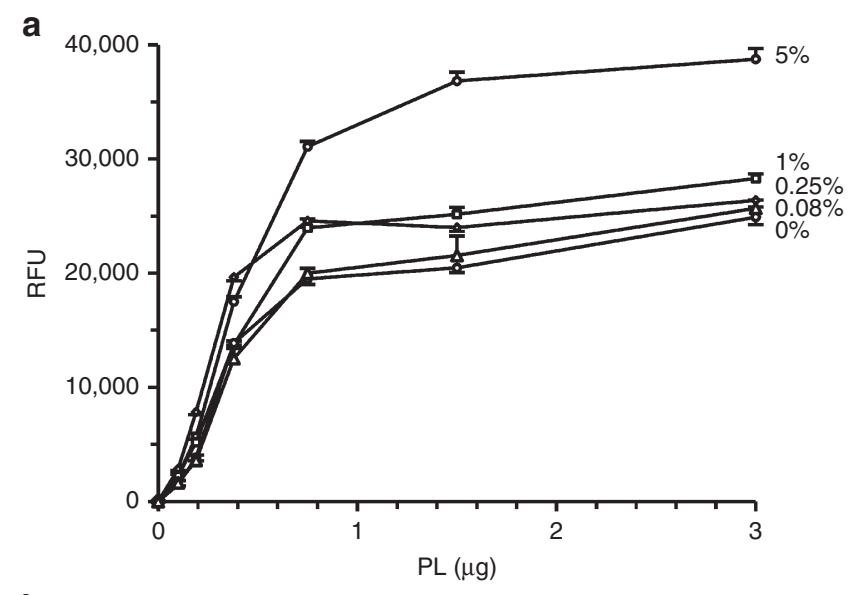

b

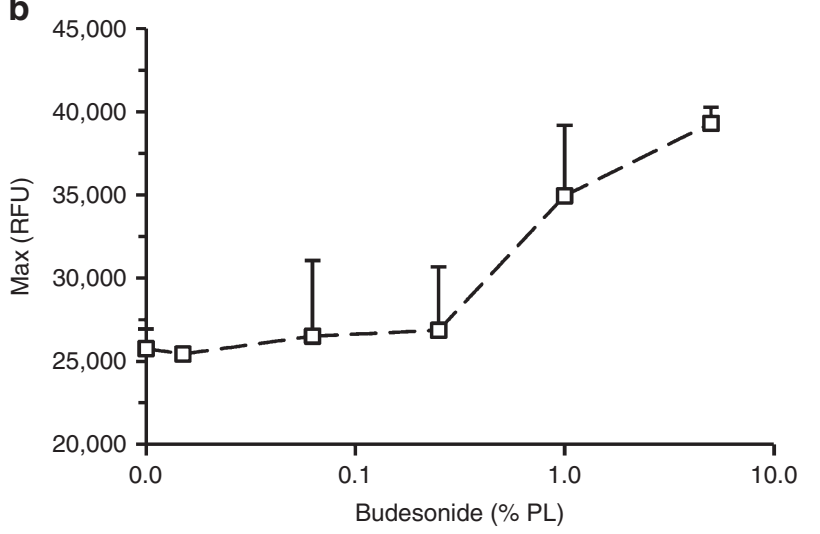

Figure 6. Budesonide addition to calfactant. (a) Representative PL doseresponse experiment for calfactant supplemented with budesonide $0-5 \%$ showing higher Max but similar $1 \frac{1}{2}$ Max with $5 \%$ budesonide (mean \pm SEM for triplicates). (b) Budesonide dose-response curve for Max film formation at $1.5 \mu \mathrm{g}$ PL. Data are mean \pm SEM for four experiments. The multimodal curve suggests a threshold effect between 1 and $5 \%$ budesonide. $\mathrm{PL}$, phospholipid.

this assay include detection of surfactant deficiencies using TA, bronchoalveolar lavage, or, potentially, amniotic fluid, assessing therapeutic surfactant quality retention under storage, testing different compounds in parallel, or testing the effect of additives.

In summary, we describe differences in in vitro film formation properties of therapeutic surfactants and demonstrate specific dose-dependent effects of SP-B for infant surfactant. The results support a critical role of SP-B for promoting and stabilizing surface film formation at lower PL concentrations. We also identified $1 / 2$ Max and tMax as new parameters for assessing film formation and homeostasis in this assay. Although the fluorescent assay has intrinsic technical limitations compared with other techniques for study of surfactant function, it may be useful for large-scale in vitro testing of film formation by artificial surfactants, evaluating effects of inhibitors and additives, and assessing the function of surfactant in clinical samples.

\section{METHODS}

\section{Patient Population and Materials}

We used TA samples obtained in a previous study from preterm infants intubated for persisting respiratory failure and randomized for late surfactant administration (day 7-21) $(1,14)$. Written, informed consent was obtained from the families of all the participating infants, and all research activities were overseen by the University of California San Francisco Committee of Human Research. Surfactant was isolated from 58 TA samples collected at different time points between 2 and $4 \mathrm{wk}$ of life from 18 intubated preterm neonates from both treatment and control groups with a gestational age of $24.9 \pm 1.1 \mathrm{wk}$ (mean $\pm \mathrm{SD}$ ). All TA samples were collected $>2 \mathrm{~d}$ after a dose of calfactant when recovery of surfactant PL and SP-B content have returned to baseline (26). For supplementation experiments, samples with low endogenous SP-B from the control group were pooled. One TA was obtained from another study involving term infants (27). Natural surfactant was obtained by bronchoalveolar lavage of adult rats as published (28). For therapeutic surfactant experiments, we used single, unexpired lots of calfactant (Infasurf; Ony, Amherst, VA), beractant (Survanta; Abbott Nutrition, Cleveland, OH), poractant alfa (Curosurf; Chiesi Farmaceutici S.p.A, Parma, Italy), colfosceryl palmitate (Exosurf; Glaxo Wellcome, Uxbridge, Middlesex, UK), lucinactant (Surfaxin; Discovery Laboratories, Warrington, PA), and budesonide inhalation suspension (Pulmocort Repsules $0.5 \mathrm{mg} / 2 \mathrm{ml}$; AstraZeneca LP, Wilmington, DE). Purified human SP-B from bronchoalveolar lavage of adult lungs, a gift of S. Hawgood, was mixed with surfactant aliquots as a $0.5 \mathrm{mg} / \mathrm{ml}$ chloroform solution followed by complete evaporation.

\section{Processing of TA Samples}

TA was centrifuged at $500 \times g$ for 5 min to remove cells. The supernatant was stored at $-80{ }^{\circ} \mathrm{C}$ in the presence of protease inhibitors and subsequently centrifuged at $27,000 \times g$ for $60 \mathrm{~min}$ to collect the large aggregate surfactant pellet, which was suspended in surfactant buffer (10 mmol/l Tris, $\left.154 \mathrm{mmol} / \mathrm{l} \mathrm{NaCl}, 1.5 \mathrm{mmol} / \mathrm{l} \mathrm{CaCl}{ }_{2}, \mathrm{pH} 7.4\right)$, assayed for PL content by phosphorus assay (29), and resuspended at a $1.5 \mathrm{mg} \mathrm{PL} / \mathrm{ml}$ concentration. Total protein was measured using QuantiPro BCA (Sigma, St Louis, MO). SP-B and SP-C fractions were isolated using Bond Elut NH2 columns (Agilent Technologies, Santa Cruz, CA) as previously described (30). SP-B in surfactant pellets was quantified using an immunodot assay and expressed as a percentage of PL by weight as previously described (1). SP-B measurements were performed 6-15 mo after TA collection and 1-3 mo before film formation studies. SP-B concentration in frozen TA samples varied $<10 \%$ over a $5-y$ period (Supplementary Methods online).

\section{Surface Film Formation Assay}

Surfactant adsorption and film formation was analyzed using the fluorescence method as previously described, with slight modifications (10). In brief, BODIPY-PC (Invitrogen Molecular Probes, Carlsbad, CA) was incubated at 3\% concentration with large aggregate surfactant $(1.5 \mathrm{mg} \mathrm{PL} / \mathrm{ml})$ at $37^{\circ} \mathrm{C}$ for $2 \mathrm{~h}$. Four serial $1: 2$ dilutions were made in surfactant buffer. Quencher consisted of Brilliant Black (Sigma-Aldrich, St Louis, MO) $6.25 \mathrm{mg} / \mathrm{ml}$ in surfactant buffer. Wells containing $100 \mu \mathrm{l}$ Brilliant Black solution received increasing amounts of labeled surfactant at the bottom, in triplicate. A Victor 3 multilabel plate reader (Perkin Elmer, Waltham, MA) was used at $37^{\circ} \mathrm{C}$ applying 3-s shake cycles with 30 -s intervals for a total of 20 cycles; excitation wavelength was $485 \mathrm{~nm}$, emission $535 \mathrm{~nm}$, counter time $0.15 /$ well. PL-related fluorescence was calculated by subtracting background (preloading) fluorescence from total (postloading) fluorescence at each time point. Results are expressed as mean/SD or SE of intraexperiment triplicates or multiple experiments, and statistical significance was assessed by linear regression, paired Student's $t$-test, or ANOVA with Bonferroni-Dunn post hoc test for multiple comparisons.

\section{SUPPLEMENTARY MATERIAL}

Supplementary material is linked to the online version of the paper at http:// www.nature.com/pr

\section{ACKNOWLEDGMENTS}

We are grateful to H.W. Taeusch for reviewing the manuscript; J.A. Clements for providing colfosceryl palmitate; Ony Inc., Amherst, VA, and Discovery Laboratories, Warrington, PA, for providing calfactant and lucinactant, respectively. 


\section{Articles $\mid$ Danhaive et al.}

\section{STATEMENT OF FINANCIAL SUPPORT}

This research was supported by National Institutes of Health (Bethesda, MD) grant PO1 HL024075.

Disclosure: The authors have no financial ties to products involved in this study and no conflicts of interest to disclose.

\section{REFERENCES}

1. Merrill JD, Ballard RA, Cnaan A, et al. Dysfunction of pulmonary surfactant in chronically ventilated premature infants. Pediatr Res 2004;56: $918-26$.

2. Hawgood S. Surfactant protein B: structure and function. Biol Neonate 2004;85:285-9.

3. Taeusch HW, Bernardino de la Serna J, Perez-Gil J, Alonso C, Zasadzinski JA. Inactivation of pulmonary surfactant due to serum-inhibited adsorption and reversal by hydrophilic polymers: experimental. Biophys J 2005;89:1769-79.

4. Polin RA, Carlo WA; Committee on Fetus and Newborn; American Academy of Pediatrics. Surfactant replacement therapy for preterm and term neonates with respiratory distress. Pediatrics 2014;133:156-63.

5. Sinha SK, Lacaze-Masmonteil T, Valls i Soler A, et al.; Surfaxin Therapy Against Respiratory Distress Syndrome Collaborative Group. A multicenter, randomized, controlled trial of lucinactant versus poractant alfa among very premature infants at high risk for respiratory distress syndrome. Pediatrics 2005;115:1030-8.

6. Sato A, Ikegami M. SP-B and SP-C containing new synthetic surfactant for treatment of extremely immature lamb lung. PLoS One 2012;7:e39392.

7. Seehase M, Collins JJ, Kuypers E, et al. New surfactant with SP-B and C analogs gives survival benefit after inactivation in preterm lambs. PLoS One 2012;7:e47631.

8. Yeh $\mathrm{TF}$, Lin $\mathrm{HC}$, Chang $\mathrm{CH}$, et al. Early intratracheal instillation of budesonide using surfactant as a vehicle to prevent chronic lung disease in preterm infants: a pilot study. Pediatrics 2008;121:e1310-8.

9. Enhorning G. Pulmonary surfactant function studied with the pulsating bubble surfactometer (PBS) and the capillary surfactometer (CS). Comp Biochem Physiol A Mol Integr Physiol 2001;129:221-6.

10. Ravasio A, Cruz A, Pérez-Gil J, Haller T. High-throughput evaluation of pulmonary surfactant adsorption and surface film formation. J Lipid Res 2008;49:2479-88.

11. Notter RH, Wang Z, Egan EA, Holm BA. Component-specific surface and physiological activity in bovine-derived lung surfactants. Chem Phys Lipids 2002;114:21-34.

12. Blanco O, Pérez-Gil J. Biochemical and pharmacological differences between preparations of exogenous natural surfactant used to treat Respiratory Distress Syndrome: role of the different components in an efficient pulmonary surfactant. Eur J Pharmacol 2007;568:1-15.

13. Ballard PL, Merrill JD, Godinez RI, Godinez MH, Truog WE, Ballard RA. Surfactant protein profile of pulmonary surfactant in premature infants. Am J Respir Crit Care Med 2003;168:1123-8.
14. Merrill JD, Ballard PL, Courtney SE, et al. Pilot trial of late booster doses of surfactant for ventilated premature infants. J Perinatol 2011;31:599-606.

15. Zuo YY, Veldhuizen RA, Neumann AW, Petersen NO, Possmayer F. Current perspectives in pulmonary surfactant-inhibition, enhancement and evaluation. Biochim Biophys Acta 2008;1778:1947-77.

16. Serrano AG, Pérez-Gil J. Protein-lipid interactions and surface activity in the pulmonary surfactant system. Chem Phys Lipids 2006;141:105-18.

17. Takamoto DY, Lipp MM, von Nahmen A, Lee KY, Waring AJ, Zasadzinski JA. Interaction of lung surfactant proteins with anionic phospholipids. Biophys J 2001;81:153-69.

18. Lopez-Rodriguez E, Echaide M, Cruz A, Taeusch HW, Perez-Gil J. Meconium impairs pulmonary surfactant by a combined action of cholesterol and bile acids. Biophys J 2011;100:646-55.

19. Krüger P, Schalke M, Wang Z, Notter RH, Dluhy RA, Lösche M. Effect of hydrophobic surfactant peptides SP-B and SP-C on binary phospholipid monolayers. I. Fluorescence and dark-field microscopy. Biophys J 1999;77:903-14.

20. Mizuno K, Ikegami M, Chen CM, Ueda T, Jobe AH. Surfactant protein-B supplementation improves in vivo function of a modified natural surfactant. Pediatr Res 1995;37:271-6.

21. Durand DJ, Clyman RI, Heymann MA, et al. Effects of a protein-free, synthetic surfactant on survival and pulmonary function in preterm lambs. J Pediatr 1985;107:775-80.

22. Guardia CG, Moya FR, Sinha S, Simmons PD, Segal R, Greenspan JS. A pharmacoeconomic analysis of in-hospital costs resulting from reintubation in preterm infants treated with lucinactant, beractant, or poractant alfa. J Pediatr Pharmacol Ther 2012;17:220-7.

23. Yu SH, McCormack FX, Voelker DR, Possmayer F. Interactions of pulmonary surfactant protein SP-A with monolayers of dipalmitoylphosphatidylcholine and cholesterol: roles of SP-A domains. J Lipid Res 1999;40:920-9.

24. Palmer D, Schürch S, Belik J. Effect of budesonide and salbutamol on surfactant properties. J Appl Physiol (1985) 2000;89:884-90.

25. King RJ, Clements JA. Surface active materials from dog lung. 3. Thermal analysis. Am J Physiol 1972;223:727-33.

26. Keller RL, Merrill JD, Black DM, et al. Late administration of surfactant replacement therapy increases surfactant protein-B content: a randomized pilot study. Pediatr Res 2012;72:613-9.

27. Cogo PE, Simonato M, Danhaive O, et al. Impaired surfactant protein B synthesis in infants with congenital diaphragmatic hernia. Eur Respir J 2013;41:677-82.

28. Subramaniam S, Bummer P, Gairola CG. Biochemical and biophysical characterization of pulmonary surfactant in rats exposed chronically to cigarette smoke. Fundam Appl Toxicol 1995;27:63-9.

29. Bartlett GR. Phosphorus assay in column chromatography. J Biol Chem 1959;234:466-8.

30. Cogo P, Baritussio A, Rosso F, et al. Surfactant-associated protein B kinetics in vivo in newborn infants by stable isotopes. Pediatr Res 2005;57:519-22. 